\title{
Assessment of Knowledge, Perception and Practices about Malaria in Some Rural Communities of South West Region, Cameroon
}

\author{
Bonaventure Tientche*, Jerome Fru-Cho, Damian Nota Anong, Hermann Ngouakam and Theresa K Nkuo-Akenji \\ Department of Microbiology and Parasitology, Faculty of Science, University of Buea, P.O.Box 63 Buea, Cameroon
}

\begin{abstract}
Background: Understanding local knowledge about malaria can help in designing sustainable community-based malaria control programs. Thus, the purpose of this study was to generate information on knowledge, perceptions and practices and the preventive measures as regards to malaria in the rural farming community.

Methods: A cross-sectional survey was conducted September to October 2013 among 851 households from 9 villages in Ekondo Titi Subdivision using structured questionnaire. Questions assessed knowledge, perceptions and practices about malaria among tribal villagers. The data were analyzed using SPSS 20.0 statistical software program.

Results: Most of the respondents $58.2 \%$ attributed malaria to mosquito bites. However, some of the respondents $(28.8 \%)$ mentioned drinking dirty water, midges and standing in the sun as the causes of malaria transmission. Avoiding stagnant water $(60.9 \%)$ and Clearing of bushes $(71.5 \%)$ were the most frequently mentioned malaria preventive measures perceived and practiced by the respondents. Only $11.05 \%$ of 851 participants owned at least one long lasting insecticide treated net (LLIN). Thirty-nine point nine $(39.9 \%)$ of respondents indicated that they experienced the last malaria episode more than three months ago. Malaria chemoprophylactic treatment was related to the educational status of the participants $(\mathrm{p}=0.001)$
\end{abstract}

Conclusions: Despite this fair knowledge and good attitudes, practices towards malaria prevention and control were poor. A considerable proportion had misconceptions about the cause and transmission of malaria suggesting the necessity of health education. To close the gap between knowledge about transmission and ownership and use of bed nets as a preventive measure, there is the need to re-energize the CHWs activities and implement the concept of night watch in the rural communities.

Keywords: Perceptions Knowledge $•$ Practice $\cdot$ Misconception • Ekondo Titi

\section{Introduction}

Despite decades of control and prevention efforts, malaria remains one of the greatest causes of morbidity and mortality in the tropical regions of the world. Globally, there are approximately 219 million clinical cases and roughly 435000 deaths due to malaria each year (WHO,2017). In Cameroon, an estimated 18 million people (about over $90 \%$ are at risk percent of the population) are at risk of infection, about $41 \%$ have at least one episode of malaria each year $[1,2]$. As recommended by the World Health Organization, Current interventions in Cameroon include, early diagnosis, artemisinin-based combination(ACT) therapies at subsidized cost for first-line treatment for uncomplicated malaria cases, intermittent preventive therapy with sulphadoxine-pyrimethamine for expectant mothers, free distribution of LLINs to pregnant women and children under five years old and the subsidizing of the cost of LLINs for other people [3], and training in the community of local health assistants capable of managing uncomplicated malaria cases and providing adequate advice to families [2].

Despite the considerable effort made to control malaria, the disease is responsible for $56 \%$ of consultations, $54 \%$ of hospital admissions and $53 \%$ of deaths among children below five years are due to malaria that in the South West Region of Cameroon [4,5]. According to the Cameroon Ministry of Public Health, $31 \%$ of consultations, $44 \%$ of hospitalizations and $18 \%$

*Address for Correspondence: Tientche B, Department of Microbiology and Parasitology, Faculty of Science, University of Buea, P.O.Box 63 Buea, Cameroon, E-mail: tientche.bonaventure@ubuea.cm

Copyright: () 2020 Tientche B, et al. This is an open-access article distributed under the terms of the Creative Commons Attribution License, which permits unrestricted use, distribution, and reproduction in any medium, provided the original author and source are credited.

Received 28 December 2019; Accepted 26 February 2020; Published 03 March 2020 of deaths occurring in health facilities are attributed to malaria [6]. Major Anopheles species incriminated include: anophele gambiae, Anophele arabiensis, Anophele funestus, Anophele Anophele moucheti, Anophele Nili [7].

However, the epidemiological pattern of the disease varies from region to region and even from time to time [8]. The prevalence of malaria parasitaemia in the Mount Cameroon region varies from $60.6 \%$, in low land altitude to $7.7 \%$ in the highlands [9]. Tchinda reported that only $52 \%$ of households possess any net, only $36 \%$ own a LLIN, and only $28 \%$ of children below 5 years were reported to have slept under a net, with $21 \%$ under a LLIN. The indoor residual spraying (IRS) is not implemented because of high transaction costs and lack of data for proper planning and decision, except few pilot operation $[10,11]$.

Deficient knowledge on malaria treatment guidelines and irrational use of antimalarials have been reported from Cameroon [3]. In a study among 1197 health service users in northern Cameroon, only 1\% identified mosquitoes as a source of transmission [12]. Geographical locations, tropical climate, and socio-economic conditions make appropriate condition for occurrence and persistent transmission of malaria in this region [13].

The practice of malaria preventive measures has been related to the level of knowledge and belief of people [14]. Social and cultural factors, although less directly studied, are also important determinants to adherence to preventive measures, therefore, can negatively influence the choice, acceptance, and use of malaria control interventions [15]. In rural Ghana, malaria treatment was often reported to be a combination of both traditional and modern methods. Treatment takes the form of self-medication at home with anti-malarial, herbal medicines and other modalities [16]. Nkuo-Akenji reported that proper health education of village mothers has proved to reduce under-five malaria morbidity and mortality significantly in Cameroon [17].

Studies about knowledge, attitudes, and practices on malaria have not 
received much attention in Cameroon. Data on the quantitative assessment of malaria burden in Cameroon rural areas where over $47 \%$ of the Cameroon population live and the community attitudes and knowledge on malaria are currently lacking. Understanding local knowledge about malaria can help in designing sustainable community-based malaria control programs that will lead to behavior change and the adoption of new ideas and technology [18].

To prepare for a successful malaria control program, it is necessary to evaluate the level of awareness, attitudes, and practices of people living in at-risk areas. This is required to get the full participation of the community in surveillance and control activities such as the use of LLINs [19].

Thus, the objective of this study is to generate information on knowledge, perceptions, and practices and the preventive measures as regards to malaria in a remote, rural, and farming community in Ekondo Titi Subdivision, South West region of Cameroon.

\section{Methods}

\section{Study population}

This study took place in Ekondo Titi Subdivision which has been described elsewhere (Tientche et al., 2016). The subdivision is made up of the maritime and the land area. The main land is composed of 26 villages. The maritime is composed of a very rich mangrove forest. The main land area is made up of an equatorial evergreen forest rich variety of fauna and floral. This forest and its biodiversity are alarmingly degrading by small holders, agro-industries like Pamol Plantations Plc (PPL) and Cameroon Development Coorporation (CDC), unsustainable illegal logging, subsistence and cash crop agriculture (palm oil and cocao) and poaching for bush meat. Slash and burn are very common farming practices in this forested area. With respect to hydrology network, the Rumpi Hills constitutes the main catchment area from which the River Meme, Ndian, and Ma'a (which are the main rivers in the Sub Division) take their rise and drain into the Atlantic Ocean. These rivers increase in volumes in the rainy season and reduce in the dry season. In addition to these main rivers there are numerous streams and springs. The sub Division also possesses a mangrove forest and creek which are breeding sites and habitat of diverse fish and other aquatic species. Poorly maintained earth roads rock the whole area and they often become very bad during the rainy seasons.

\section{Participant selection and data collection}

This was a household-based study that involved face-to-face interviews with heads of households. Investigations were conducted in 09 communities including Kitta Balue, Ngolo Metoko, Masore Balue, Bongongo Barombi I, Bogongo Barombi II, Bekora Barombi, Lipenja Barombi, Lipenja Camp, Kumbe Balondo. Sites selected were rural, isolated, typically enclaved and largely covered by tropical rainforests with diverse ethnic groups.

The house of the village chief was considered as the starting-point and subsequently all participants who consented to the study. A household was defined as the entity in which people live together and have a meal from a common cooking facility, and a householder was defined as the person who is perceived by members of the household as the key decision-maker in the family (Ahmed et al., 2009) [20]. Parents (household head or his spouse) were interviewed using a structured questionnaire. Interviews were conducted in private to reduce the influence of other people. The questionnaires were constructed in English and interviews were conducted in the local language and in pidgin through the help of community health workers. The questionnaire was in a closed-ended format, with some questions allowing open-ended answers.

\section{Ethical consideration}

The study obtained ethical clearance from the Regional Delegation of Public Health, Buea. A supportive letter was obtained from the District Health Officer at Ekondo Titi before data collection and written informed consent was obtained from voluntary participants during data collection.
The respondents were assured of their right to withdraw from the interview at any time they would wish during the exercise.

\section{Data analysis}

Once recorded, the data were entered in an Excel database and exported to SPSS 20.0 for analysis. Pearson chi-square was applied to compare proportions. The association between variables was assessed using 95\% Confidence Intervals $(\mathrm{Cl})$. In addition to textual descriptions, results were summarized in the form of tables and figures

\section{Results}

\section{Baseline characteristics of respondents}

A total of 851 household's heads were interviewed, including 371 (43.6\%) females and 480 (56.4\%) males Table 1 . About 122 (14.3\%) did not acquire any formal education, and the majority of participants $562(66.0 \%)$ completed primary school education, while only $1.8 \%$ had attended tertiary education. The highest illiteracy rate $17(32.7 \%)$ was observed in Masore Balue Figure 1. Detailed socio-demographic characteristics are presented in Table 1. A total of $602(70.7 \%)$ of the respondents were married while a negligible number 25 (2.9\%) being divorced. The economic activity carried by the vast majority of the respondents was farming 438 (51.5\%) while a good number, 276((32.4\%) were working as laborers in Pamol Plantations Ltd. With regard to education level and sex of the population with no formal education by villages, women accounted for $54.9 \%$ while nearly $60 \%$ of males have completed primary school respectively Table 2 .

\section{Knowledge of malaria transmission}

When asked 'How does a person get malaria? The majority of respondents $449(58.2 \%)$ correctly identified mosquitoes as transmitting malaria, while $207(26.8 \%)$ did not identify any mechanism for malaria transmission Table 3 . Even though, a majority of respondents were aware of the cause of malaria, a sizable proportion 177 (28.8\%) still had misconceptions, such as drinking dirty water, overwork and walk and standing in the sun as the causes of malaria transmission Table 3. Less frequently mentioned causes were exposure to fire, poor environment, or eating a specific food such as cassava semolina (water fufu*). Correct knowledge of respondents regarding the transmission of malaria was significantly associated with participant's community $(p=0.001)$ Table 3 . Bogongo Barombi II and Ngolo Metoko communities were found better in identifying the cause of malaria than those participants from Bekora Barombi and Kitta Balue $(p=0.01)$. The study revealed no gender $(\chi 2=0.58$; $p=0.0809)$ Table 4 nor levels of education $(\alpha 2=8.811 ; p=0.066)$ Table 5 significant differences in respondents' $s$ knowledge of malaria transmission by mosquito bite.

\section{Knowledge of prevention}

Pertaining to the methods of preventing malaria Table 6 , avoiding stagnant water 507(60.9\%) and Clearing of bushes 596(71.5\%) were reported as the primary means. Only $93(11.05 \%)$ of respondents acknowledged the usage of net as a barrier to mosquito bite. Among those who believed that malaria is preventable, $98(11.8 \%)$ reported residual indoor house spraying, 183(22.0\%) mentioned the usage of mosquito coil and $84(10.1 \%)$ indicated chemoprophylactic treatment. Concomitantly, a high number of participants 392(47.1\%) acknowledged the use of traditional medicine. Knowledge of respondents about preventive measures was significantly different among various communities $(p<0.05)$ Table 6 . Table 7 demonstrated that household heads with higher educational status were more likely to use chemoprophylactic treatment to avoid malaria $(p=0.001)$.

\section{Household use of LLINs}

Ninety-three households 93(11.05\%) surveyed owned LLINs and all of these were given to them freely when a household member delivered a baby. The usage of net varied significantly among various communities $(P=0.001)$ Table 8 and was not significantly associated with the level of 
Table 1. Socio-demographic characteristics of all respondents by communities.

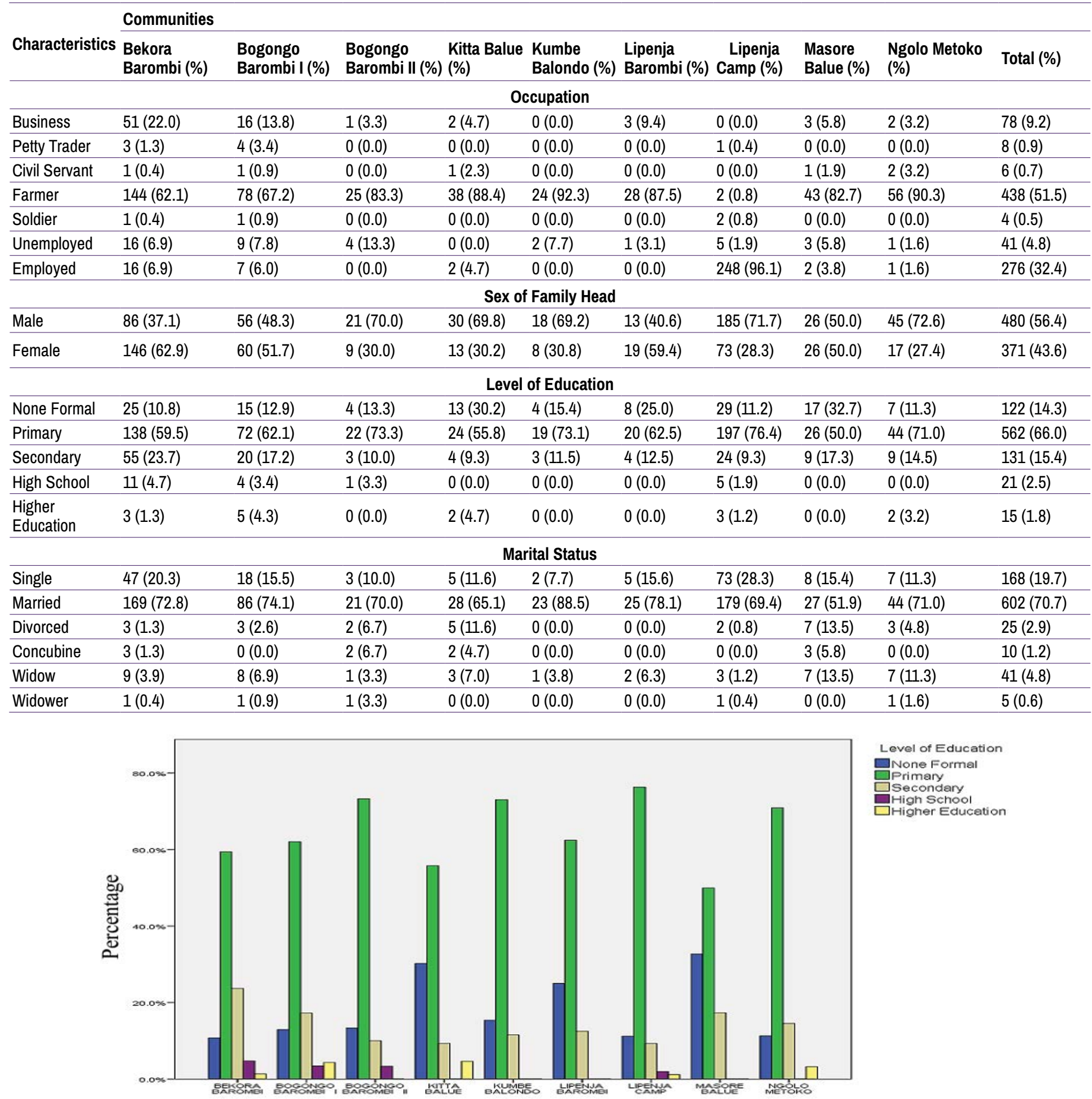

Level of Education

Figure 1. Level of education in each community.

Table 2. Level of education by gender in each community.

\begin{tabular}{|c|c|c|c|c|c|c|c|c|c|c|}
\hline \multirow[b]{2}{*}{$\begin{array}{l}\text { Level of } \\
\text { Education }\end{array}$} & \multicolumn{10}{|l|}{ Community } \\
\hline & $\begin{array}{l}\text { Bekora } \\
\text { Barombi (\%) }\end{array}$ & $\begin{array}{l}\text { Bogongo } \\
\text { Barombi } \\
\text { I (\%) }\end{array}$ & $\begin{array}{l}\text { Bogongo } \\
\text { Barombi li (\%) }\end{array}$ & $\begin{array}{l}\text { Kitta Balue } \\
(\%)\end{array}$ & $\begin{array}{l}\text { Kumbe } \\
\text { Balondo (\%) }\end{array}$ & $\begin{array}{l}\text { Lipenja } \\
\text { Barombi (\%) }\end{array}$ & $\begin{array}{c}\text { Lipenja } \\
\text { Camp (\%) }\end{array}$ & $\begin{array}{l}\text { Masore } \\
\text { Balue (\%) }\end{array}$ & $\begin{array}{l}\text { Ngolo Metoko } \\
(\%)\end{array}$ & Total (\%) \\
\hline \multicolumn{11}{|c|}{ None Formal } \\
\hline Male & $5(20.0)$ & $6(40.0)$ & $1(25.0)$ & $8(61.5)$ & $3(75.0)$ & $1(12.5)$ & $18(62.1)$ & $11(64.7)$ & $2(28.6)$ & 55 (45.1) \\
\hline Female & $20(80.0)$ & $9(60.0)$ & $3(75.0)$ & $5(38.5)$ & $1(25.0)$ & $7(87.5)$ & $11(37.9)$ & $6(35.3)$ & $5(71.4)$ & $67(54.9)$ \\
\hline \multicolumn{11}{|c|}{ Primary } \\
\hline Male & 57 (41.3) & $34(47.2)$ & $16(72.7)$ & $19(79.2)$ & $13(68.4)$ & $9(45.0)$ & $141(71.6)$ & $11(42.3)$ & $34(77.3)$ & 334 (59.4) \\
\hline
\end{tabular}




\begin{tabular}{lccllllllll}
\hline Female & $81(58.7)$ & $38(52.8)$ & $6(27.3)$ & $5(20.8)$ & $6(31.6)$ & $11(55.0)$ & $56(28.4)$ & $15(57.7)$ & $10(22.7)$ & $228(40.6)$ \\
\hline Male & $20(36.4)$ & $9(45.0)$ & $3(100.0)$ & $2(50.0)$ & $2(66.7)$ & $3(75.0)$ & $20(83.3)$ & $4(44.4)$ & $7(77.8)$ & $70(53.4)$ \\
\hline Female & $35(63.6)$ & $11(55.0)$ & $0(0.0)$ & $2(50.0)$ & $1(33.3)$ & $1(25.0)$ & $4(16.7)$ & $5(55.6)$ & $2(22.2)$ & $61(46.6)$ \\
\hline \multicolumn{7}{c}{ High School } \\
\hline Male & $3(27.3)$ & $4(100.0)$ & $1(100.0)$ & $0(0.0)$ & $0(0.0)$ & $0(0.0)$ & $3(60.0)$ & $0(0.0)$ & $0(0.0)$ & $11(52.4)$ \\
\hline Female & $8(72.7)$ & $0(0.0)$ & $0(0.0)$ & $0(0.0)$ & $0(0.0)$ & $0(0.0)$ & $2(40.0)$ & $0(0.0)$ & $0(0.0)$ & $10(47.6)$ \\
\hline Male & $1(33.3)$ & $3(60.0)$ & $0(0.0)$ & $1(50.0)$ & $0(0.0)$ & $0(0.0)$ & $3(100.0)$ & $0(0.0)$ & $2(100.0)$ & $10(66.7)$ \\
\hline Female & $2(66.7)$ & $2(40.0)$ & $0(0.0)$ & $1(50.0)$ & $0(0.0)$ & $0(0.0)$ & $0(0.0)$ & $0(0.0)$ & $0(0.0)$ & $5(33.3)$ \\
\hline
\end{tabular}

Table 3. Knowledge and attitude of respondents regarding the transmission of malaria by community.

\begin{tabular}{|c|c|c|c|c|c|c|c|c|c|c|c|c|}
\hline $\begin{array}{l}\text { How is Malaria } \\
\text { Transmitted }\end{array}$ & $\begin{array}{l}\text { Bekora } \\
\text { Barombi n } \\
\text { (\%) }\end{array}$ & $\begin{array}{l}\text { Bogongo } \\
\text { Barombi I } \\
\text { n (\%) }\end{array}$ & $\begin{array}{l}\text { Bogongo } \\
\text { Barombi II } \\
\text { i (\%) }\end{array}$ & $\begin{array}{l}\text { Kitta } \\
\text { Balue n } \\
\text { (\%) }\end{array}$ & $\begin{array}{l}\text { Kumbe } \\
\text { Balondo n } \\
\text { (\%) }\end{array}$ & $\begin{array}{l}\text { Lipenja } \\
\text { Barombi ( } \\
\text { n\%) }\end{array}$ & $\begin{array}{l}\text { Lipenja } \\
\text { Camp n (\%) }\end{array}$ & $\begin{array}{l}\text { Masore } \\
\text { Balue n } \\
(\%)\end{array}$ & $\begin{array}{l}\text { Ngolo } \\
\text { Metoko n } \\
\text { (\%) }\end{array}$ & Total n (\%) & $\begin{array}{l}\text { Chi } \\
\text { square }\end{array}$ & P-Value \\
\hline $\begin{array}{l}\text { Blood } \\
\text { Transmission }\end{array}$ & $0(0.0)$ & $0(0.0)$ & $0(0.0)$ & $1(2.3)$ & $0(0.0)$ & $0(0.0)$ & $0(0.0)$ & $0(0.0)$ & $0(0.0)$ & $1(0.1)$ & 18.813 & 0.016 \\
\hline Congenital & $0(0.0)$ & $0(0.0)$ & $0(0.0)$ & $0(0.0)$ & $0(0.0)$ & $0(0.0)$ & $0(0.0)$ & $0(0.0)$ & $0(0.0)$ & $0(0.0)$ & & \\
\hline Mosquito Bite & 98 (44.1) & $63(60.0)$ & $26(86.7)$ & $23(53.5)$ & 19 (79.2) & $24(77.4)$ & $131(58.5)$ & $27(55.1)$ & $38(86.4)$ & $449(58.2)$ & 37.147 & 0.001 \\
\hline $\begin{array}{l}\text { Infected piercing } \\
\text { Objects }\end{array}$ & $0(0.0)$ & $0(0.0)$ & $0(0.0)$ & $0(0.0)$ & $0(0.0)$ & $0(0.0)$ & $0(0.0)$ & $0(0.0)$ & $0(0.0)$ & $0(0.0)$ & & \\
\hline All of the above & $4(1.8)$ & $0(0.0)$ & $0(0.0)$ & $0(0.0)$ & $0(0.0)$ & $0(0.0)$ & $0(0.0)$ & $0(0.0)$ & $0(0.0)$ & $4(0.5)$ & 10.723 & 0.218 \\
\hline I Don't Know & $66(29.7)$ & $30(28.6)$ & $4(13.3)$ & 16 (37.2) & $4(16.7)$ & $10(32.3)$ & $58(25.9)$ & $19(38.8)$ & $0(0.0)$ & $207(26.8)$ & 34.722 & 0.001 \\
\hline Midges Bite & $7(3.2)$ & 14 (13.3) & $7(23.3)$ & $6(14.0)$ & $12(50.0)$ & $1(3.2)$ & $1(0.4)$ & $3(6.1)$ & $13(29.5)$ & $64(8.3)$ & 115.46 & 0.001 \\
\hline Food & $4(1.8)$ & $0(0.0)$ & $0(0.0)$ & $0(0.0)$ & $0(0.0)$ & $0(0.0)$ & $0(0.0)$ & $0(0.0)$ & $0(0.0)$ & $4(0.5)$ & 10.448 & 0.218 \\
\hline $\begin{array}{l}\text { Contaminated } \\
\text { Water }\end{array}$ & $28(12.6)$ & $5(4.8)$ & $1(3.3)$ & $0(0.0)$ & $0(0.0)$ & $0(0.0)$ & 39 (17.4) & $0(0.0)$ & $0(0.0)$ & $73(9.5)$ & 41.558 & 0.001 \\
\hline Over Work & $16(7.2)$ & $6(5.7)$ & $2(6.7)$ & $0(0.0)$ & $1(4.2)$ & $2(6.5)$ & $6(2.7)$ & $1(2.0)$ & $7(15.9)$ & $14(5.3)$ & 14.923 & 0.061 \\
\hline Water Fufu & $2(0.9)$ & $0(0.0)$ & $0(0.0)$ & $1(2.3)$ & $0(0.0)$ & $0(0.0)$ & $3(1.3)$ & $2(4.1)$ & $0(0.0)$ & $8(1.0)$ & 8.279 & 0.407 \\
\hline Fever & $1(0.5)$ & $0(0.0)$ & $0(0.0)$ & $0(0.0)$ & $0(0.0)$ & $0(0.0)$ & $0(0.0)$ & $0(0.0)$ & $0(0.0)$ & $1(0.1)$ & 2.671 & 0.953 \\
\hline Dirtness & $2(0.9)$ & $0(0.0)$ & $0(0.0)$ & $0(0.0)$ & $0(0.0)$ & $0(0.0)$ & $1(0.4)$ & $2(4.1)$ & $0(0.0)$ & $5(0.6)$ & 11.756 & 0.162 \\
\hline $\begin{array}{l}\text { Poor } \\
\text { Environment }\end{array}$ & $12(5.4)$ & $0(0.0)$ & $0(0.0)$ & $0(0.0)$ & $0(0.0)$ & $0(0.0)$ & $14(6.3)$ & $1(2.0)$ & $0(0.0)$ & $27(3.5)$ & 17.674 & 0.024 \\
\hline Fire & $1(0.5)$ & $4(3.8)$ & $0(0.0)$ & $0(0.0)$ & $1(4.2)$ & $0(0.0)$ & $1(0.4)$ & $0(0.0)$ & $0(0.0)$ & $7(0.9)$ & 15.568 & 0.049 \\
\hline Sun & 26 (11.7) & $9(8.6)$ & $2(6.7)$ & $1(2.3)$ & $0(0.0)$ & $0(0.0)$ & $1(0.4)$ & $1(2.0)$ & $0(0.0)$ & $40(5.2)$ & 42.676 & 0.001 \\
\hline
\end{tabular}

Table 4. Knowledge of respondents about the transmission of malaria by gender.

\begin{tabular}{|c|c|c|c|c|c|}
\hline \multirow{2}{*}{ How is Malaria Transmitted } & \multicolumn{5}{|c|}{ Sex of Family Head } \\
\hline & Male n (\%) & Female n (\%) & Total n (\%) & Statistical test & P-value \\
\hline Blood Transmission & $1(0.2)$ & $0(0.0)$ & $1(0.1)$ & Fisher's exact & 1.000 \\
\hline Congenital & $0(0.0)$ & $0(0.0)$ & $0(0.0)$ & & \\
\hline Mosquito Bite & $255(59.4)$ & $194(56.6)$ & 449 (58.2) & $X^{2}=0.58$ & 0.809 \\
\hline Infected piercing objects & $0(0.0)$ & $0(0.0)$ & $0(0.0)$ & & \\
\hline All of the above & $0(0.0)$ & $4(1.2)$ & $4(0.5)$ & Fisher's exact & 0.036 \\
\hline I Don't Know & $114(26.6)$ & $93(27.1)$ & $207(26.8)$ & $X^{2}=0.197$ & 0.657 \\
\hline Midges Bite & $40(9.3)$ & $24(7.0)$ & $64(8.3)$ & $X^{2}=1.046$ & 0.306 \\
\hline Food & $1(0.2)$ & $3(0.9)$ & $4(0.5)$ & Fisher's exact & 0.323 \\
\hline Water & 48 (11.2) & $25(7.3)$ & $73(9.5)$ & $X^{2}=2.838$ & 0.092 \\
\hline Over Work & $21(4.9)$ & $20(5.8)$ & $41(5.3)$ & $X^{2}=0.471$ & 0.493 \\
\hline Water Fufu & $6(1.4)$ & $2(0.6)$ & $8(1.0)$ & Fisher's exact & 0.477 \\
\hline Fever & $1(0.2)$ & $0(0.0)$ & $1(0.1)$ & Fisher's exact & 1.000 \\
\hline Dirt & $2(0.5)$ & $3(0.9)$ & $5(0.6)$ & Fisher's exact & 0.658 \\
\hline Poor Environment & $11(2.6)$ & $16(4.7)$ & $27(3.5)$ & $X^{2}=2.782$ & 0.095 \\
\hline Fire & $3(0.7)$ & $4(1.2)$ & $7(0.9)$ & Fisher's exact & 0.477 \\
\hline Sun & $12(2.8)$ & $28(8.2)$ & $40(5.2)$ & $X^{2}=11.900$ & 0.001 \\
\hline
\end{tabular}

education in various communities $\left(\chi^{2}=7.533 p=0.110\right)$ Table 9 . The use of nets on doors was found in 34(4.04\%) respondents, who used them not only for a physical barrier against mosquito bite but also for malaria prevention. The use of the net on windows was very low at $13(1.54 \%)$.

\section{Period of last malaria episode}

The Period of last malaria episode among the respondents is presented in Figure 2. Thirty-nine point nine $(39.9 \%)$ of participants indicated that they experienced the last malaria episode more than three months ago, 
Table 5. Knowledge of respondents about malaria transmission by Level of education.

\begin{tabular}{|c|c|c|c|c|c|c|c|c|}
\hline \multicolumn{9}{|l|}{ Level of Education } \\
\hline $\begin{array}{l}\text { Knowledge of Malaria } \\
\text { Transmission }\end{array}$ & None Formal & Primary & Secondary & High School & Higher Education & Total & $\begin{array}{l}\text { Chi square } \\
\text { value }\end{array}$ & P-value \\
\hline Blood Transmission & $1(0.9)$ & $0(0.0)$ & $0(0.0)$ & $0(0.0)$ & $0(0.0)$ & $1(0.1)$ & 5.982 & 0.200 \\
\hline Congenital & $0(0.0)$ & $0(0.0)$ & $0(0.0)$ & $0(0.0)$ & $0(0.0)$ & $0(0.0)$ & & \\
\hline Mosquito Bite & $59(55.1)$ & $296(58.3)$ & $70(56.9)$ & $14(70.0)$ & $10(71.4)$ & $449(58.2)$ & 3.767 & 0.439 \\
\hline Infected Piercing Objects & $0(0.0)$ & $0(0.0)$ & $0(0.0)$ & $0(0.0)$ & $0(0.0)$ & $0(0.0)$ & & \\
\hline All of the above & $0(0.0)$ & $2(0.4)$ & $2(1.6)$ & $0(0.0)$ & $0(0.0)$ & $4(0.5)$ & 4.029 & 0.402 \\
\hline I Don't Know & $35(32.7)$ & $137(27.0)$ & $30(24.4)$ & $2(10.0)$ & $3(21.4)$ & $207(26.8)$ & 4.059 & 0.398 \\
\hline Midges Bite & $7(6.5)$ & $45(8.9)$ & $10(8.1)$ & $1(5.0)$ & $1(7.1)$ & $64(8.3)$ & 0.997 & 0.910 \\
\hline Food & $1(0.9)$ & $2(0.4)$ & $1(0.8)$ & $0(0.0)$ & $0(0.0)$ & $4(0.5)$ & 0.886 & 0.927 \\
\hline Water & $15(14.0)$ & $46(9.1)$ & $9(7.3)$ & $3(15.0)$ & $0(0.0)$ & $73(9.5)$ & 5.027 & 0.285 \\
\hline Over Work & $6(5.6)$ & $29(5.7)$ & $5(4.1)$ & $1(5.0)$ & $0(0.0)$ & $41(5.3)$ & 1.192 & 0.879 \\
\hline Water Fufu & $3(2.8)$ & $3(0.6)$ & $2(1.6)$ & $0(0.0)$ & $0(0.0)$ & $8(1.0)$ & 4.844 & 0.304 \\
\hline Fever & $0(0.0)$ & $0(0.0)$ & $1(0.8)$ & $0(0.0)$ & $0(0.0)$ & $1(0.1)$ & 5.503 & 0.239 \\
\hline Dirt & $1(0.9)$ & $4(0.8)$ & $0(0.0)$ & $0(0.0)$ & $0(0.0)$ & $5(0.6)$ & 1.248 & 0.870 \\
\hline Poor Environment & $5(4.7)$ & $13(2.6)$ & $8(6.5)$ & $1(5.0)$ & $0(0.0)$ & $27(3.5)$ & 6.027 & 0.197 \\
\hline Fire & $2(1.9)$ & $5(1.0)$ & $0(0.0)$ & $0(0.0)$ & $0(0.0)$ & $7(0.9)$ & 2.414 & 0.660 \\
\hline Sun & $5(4.7)$ & $25(4.9)$ & $8(6.5)$ & $2(10.0)$ & $0(0.0)$ & $40(5.2)$ & 2.587 & 0.629 \\
\hline
\end{tabular}

Table 6. Knowledge of respondents about preventive measures used by communities.

\begin{tabular}{|c|c|c|c|c|c|c|c|c|c|c|c|c|}
\hline \multirow[b]{2}{*}{$\begin{array}{l}\text { Preventive } \\
\text { Measures }\end{array}$} & \multicolumn{12}{|c|}{ Community } \\
\hline & $\begin{array}{c}\text { Bekora } \\
\text { Barombi } \\
(\%)\end{array}$ & $\begin{array}{c}\text { Bogongo } \\
\text { Barombi } \\
\text { I (\%) }\end{array}$ & $\begin{array}{c}\text { Bogongo } \\
\text { Barombi li } \\
(\%)\end{array}$ & $\begin{array}{l}\text { Kitta Balue } \\
(\%)\end{array}$ & $\begin{array}{c}\text { Kumbe } \\
\text { Balondo } \\
\text { (\%) }\end{array}$ & $\begin{array}{l}\text { Lipenja } \\
\text { Barombi } \\
\text { (\%) }\end{array}$ & $\begin{array}{l}\text { Lipenja } \\
\text { Camp (\%) }\end{array}$ & $\begin{array}{c}\text { Masore } \\
\text { Balue (\%) }\end{array}$ & $\begin{array}{l}\text { Ngolo } \\
\text { Metoko } \\
(\%)\end{array}$ & Total (\%) & $\begin{array}{l}\text { Chi } \\
\text { square }\end{array}$ & P-value \\
\hline Spray & 27 (11.6) & $15(13.0)$ & $2(6.7)$ & $6(15.8)$ & $2(8.3)$ & $2(6.3)$ & $39(15.7)$ & $1(2.0)$ & $4(6.5)$ & 98 (11.8) & 11.957 & 0.153 \\
\hline $\begin{array}{l}\text { Avoiding Stagnant } \\
\text { Water }\end{array}$ & $154(66.4)$ & $85(73.9)$ & $28(93.3)$ & $19(50.0)$ & $12(50.0)$ & $30(93.8)$ & $113(45.4)$ & $29(56.9)$ & $37(59.7)$ & $507(60.9)$ & 76.368 & 0.001 \\
\hline Clearing of Bushes & $186(80.2)$ & $92(80.0)$ & $29(96.7)$ & $23(60.5)$ & $22(91.7)$ & $30(93.8)$ & $115(46.2)$ & $47(92.2)$ & $52(83.9)$ & $596(71.5)$ & 138.691 & 0.001 \\
\hline $\begin{array}{l}\text { Use of Traditional } \\
\text { Medicine }\end{array}$ & $132(56.9)$ & $50(43.5)$ & $15(50.0)$ & $4(10.5)$ & $13(54.2)$ & $12(37.5)$ & $109(43.8)$ & 24 (47.1) & 33 (53.2) & $392(47.1)$ & 38.842 & 0.001 \\
\hline $\begin{array}{l}\text { Chemoprophylactic } \\
\text { treatment }\end{array}$ & $25(10.8)$ & $17(14.8)$ & $3(10.0)$ & $0(0.0)$ & $6(25.0)$ & $14(43.8)$ & $11(4.4)$ & $1(2.0)$ & 7 (11.3) & 84 (10.1) & 67.241 & 0.001 \\
\hline Use of Coil & $23(9.9)$ & $30(26.1)$ & 7 (23.3) & $10(26.3)$ & $5(20.8)$ & $3(9.4)$ & $81(32.5)$ & $20(39.2)$ & $4(6.5)$ & $183(22.0)$ & 54.909 & 0.001 \\
\hline
\end{tabular}

Table 7. Relationship between Preventive measures and levels of education.

\begin{tabular}{|c|c|c|c|c|c|c|c|c|}
\hline Preventive Measures & $\begin{array}{l}\text { None Formal } \\
\mathrm{n}(\%)\end{array}$ & $\begin{array}{l}\text { Primary } \\
\mathrm{n}(\%)\end{array}$ & $\begin{array}{l}\text { Secondary } \\
\mathrm{n}(\%)\end{array}$ & $\begin{array}{l}\text { High School } \\
\mathrm{nl}(\%)\end{array}$ & $\begin{array}{l}\text { Higher Education } \\
\text { n (\%) }\end{array}$ & $\begin{array}{l}\text { Total } \\
\text { n (\%) }\end{array}$ & Chi square & P-value \\
\hline Spray & $11(9.5)$ & $63(11.4)$ & $18(13.8)$ & $5(23.8)$ & $1(6.7)$ & $98(11.8)$ & 4.897 & 0.298 \\
\hline Avoiding Stagnant Water & $66(56.9)$ & $327(59.3)$ & $87(66.9)$ & $17(81.0)$ & $10(66.7)$ & $507(60.9)$ & 8.811 & 0.066 \\
\hline Clearing of Bushes & $84(72.4)$ & $381(69.1)$ & $102(78.5)$ & $17(81.0)$ & $12(80.0)$ & $596(71.5)$ & 7.154 & 0.128 \\
\hline Use of Traditional Medicine & $61(52.6)$ & $262(47.5)$ & $53(40.8)$ & $11(52.4)$ & $5(33.3)$ & $392(47.1)$ & 3.803 & 0.433 \\
\hline Chemoprophylactic treatment & $10(8.6)$ & $47(8.5)$ & $14(10.8)$ & $9(42.9)$ & $4(26.7)$ & $84(10.1)$ & 32.360 & 0.001 \\
\hline Use of Coil & $24(20.7)$ & $124(22.5)$ & $23(17.7)$ & $6(28.6)$ & $6(40.0)$ & $183(22.0)$ & 5.217 & 0.266 \\
\hline
\end{tabular}

Table 8. Use of nets in various communities.

Community

\begin{tabular}{|c|c|c|c|c|c|c|c|c|c|c|c|c|}
\hline Use of nets & & $\begin{array}{l}\text { Bogongo } \\
\text { Barombi Inl } \\
\text { (\%) }\end{array}$ & $\begin{array}{l}\text { Bogongo } \\
\text { Barombi li } \\
\text { nli (\%) }\end{array}$ & $\begin{array}{l}\text { Kitta Balue } \\
\text { n (\%) }\end{array}$ & $\begin{array}{l}\text { Kumbe } \\
\text { Balondo n } \\
\text { (\%) }\end{array}$ & $\begin{array}{l}\text { Lipenja } \\
\text { Barombi n } \\
\text { (\%) }\end{array}$ & $\begin{array}{l}\text { Lipenja } \\
\text { Camp n (\%) }\end{array}$ & $\begin{array}{l}\text { Masore } \\
\text { Balue } \mathrm{n}(\%)\end{array}$ & $\begin{array}{l}\text { Ngolo } \\
\text { Metoko n } \\
\text { (\%) }\end{array}$ & Total (\%) & $\begin{array}{l}\text { Chi } \\
\text { square }\end{array}$ & P-value \\
\hline $\begin{array}{l}\text { Ownership of } \\
\text { LLINs }\end{array}$ & 38 (16.9) & 13 (11.2) & $6(20.7)$ & $0(0.0)$ & $1(3.8)$ & $1(3.1)$ & $32(12.5)$ & $2(3.8)$ & $0(0.0)$ & 93 (11.05) & 29.107 & 0.001 \\
\hline $\begin{array}{l}\text { Use of } \\
\text { Window } \\
\text { screens }\end{array}$ & $4(1.8)$ & $4(3.4)$ & $0(0.0)$ & $2(4.7)$ & $0(0.0)$ & $0(0.0)$ & $3(1.2)$ & $0(0.0)$ & $0(0.0)$ & $13(1.54)$ & 9.055 & 0.338 \\
\hline $\begin{array}{l}\text { Use of Door } \\
\text { screens }\end{array}$ & $27(12.0)$ & $4(3.4)$ & $0(0.0)$ & $1(2.3)$ & $0(0.0)$ & $0(0.0)$ & $2(0.8)$ & $0(0.0)$ & $0(0.0)$ & $34(4.04)$ & 51.113 & 0.001 \\
\hline None & $157(69.8)$ & $96(82.8)$ & $23(79.3)$ & $40(93.0)$ & $25(96.2)$ & 31 (96.9) & $233(90.7)$ & 50 (96.2) & $62(100.0)$ & $718(85.27)$ & 85.608 & 0.001 \\
\hline
\end{tabular}


Table 9. Relationship between the Use of LLINs and the level of education in various communities.

\begin{tabular}{|c|c|c|c|c|c|c|c|c|}
\hline \multirow[b]{2}{*}{ Use of nets } & \multicolumn{8}{|c|}{ Level of Education } \\
\hline & $\begin{array}{l}\text { None Formal } \\
(\%)\end{array}$ & Primary (\%) & Secondary (\%) & High School (\%) & $\begin{array}{l}\text { Higher Education } \\
\text { (\%) }\end{array}$ & Total (\%) & $x^{2}$ & P-value \\
\hline Use of LLINs & $12(9.9)$ & $57(10.3)$ & $16(12.4)$ & $6(28.6)$ & $2(13.3)$ & 93 (11.05) & 7.533 & 0.110 \\
\hline $\begin{array}{l}\text { Use of Window } \\
\text { screens }\end{array}$ & $1(0.8)$ & $11(2.0)$ & $1(0.8)$ & $0(0.0)$ & $0(0.0)$ & $13(1.54)$ & 2.163 & 0.706 \\
\hline $\begin{array}{l}\text { Use of Door } \\
\text { screens }\end{array}$ & $1(0.8)$ & $24(4.3)$ & $5(3.9)$ & $1(4.8)$ & $3(20.0)$ & $34(4.04)$ & 13.379 & 0.010 \\
\hline None & $109(90.1)$ & $477(85.8)$ & $108(83.7)$ & $14(66.7)$ & $10(66.7)$ & $718(85.3)$ & 11.324 & 0.023 \\
\hline
\end{tabular}

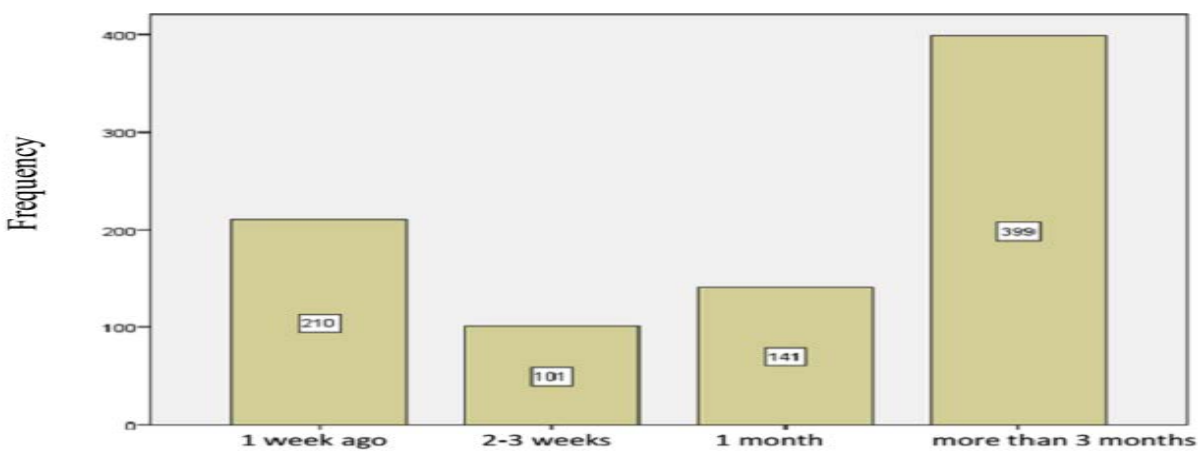

Figure 2. Frequency of malaria episode.

$21 \%$ contracted malaria less than 1 weeks ago while $10 \%$ of participants acknowledged to have had a bout of malaria two to three weeks ago.

\section{Discussion}

The current study was carried out in 9 villages in Ekondo Titi Subdivision, South West Region, Cameroon to provide baseline information about malaria-related knowledge, practices, and behavior to be used in the development of community health education in order to increase community participation in the prevention and control of malaria. Understanding community perception about malaria and the underlying intervention for its management has a policy implication for mounting successful prevention and control initiatives [19].

In this endemic area, people are aware of the role of mosquito bites in the transmission of malaria. The majority of the respondents $470(58.2 \%)$ mentioned that mosquito bite was the possible cause of malaria. Our result is lower than those reported in some sub-Saharan countries $[21,22]$ but higher than that reported by Eyobo [23] in South Sudan and Aikpon (2015) in Ethiopia. These are not encouraging results when compared to 82\% [2] and $88 \%$ [14] reported in other parts of Cameroon. The difference might be due to the lack of house-to-house health extension services, the absence of health education, communication and electricity to get access to information broadcast by media houses. The correct knowledge of transmission of malaria by mosquito bite was not found significantly associated with gender $\left(\chi^{2}=0.58, p=0.809\right)$.

Twenty point eight percent (28.8\%) of respondents had misconception regarding the mode of transmission of malaria such as midges, drinking dirty water, standing or walking in the sun. Other misconceptions including; fire, eating contaminated food, fever, overwork, poor environment. This finding is consistent with the study [24] (29\%), Ndo 2011(18\%) despite considerable efforts deployed by the Cameroon government for malaria control. Likewise, misconceptions have been reported in other studies in Nigeria and in Ethiopia $[25,26]$. Poor nutrition and alcohol consumption can compromise a person's immune status and this may explain why few participants ascribed malaria to food and alcohol consumption [27]. It is critical to correct such misconceptions through simple health education messages if the NMCP (National Malaria Control Programme) has to sustain and increase its successes.
Interestingly, there was a significant difference between gender as regard to the exposure to the sun in the transmission of malaria $(p=0.001)$. As the primary caregivers at home, this belief regarding the role of the sun in malaria transmission may be detrimental to children's health as their mother might be reluctant to use ITN as a preventive method. Nkuo-Akenji indicated that proper health education of villagers, particularly mothers is an important strategy that would reduce malaria morbidity and mortality significantly. Consequently, Health education needs to be context-sensitive, i.e. mindful of how knowledge will ultimately be transformed into action [28].

The low literacy level could also have played a significant role in this level of awareness. This could be understood by the high illiteracy rate 684 $(80.3 \%)$ observed in these communities.

Comparatively, respondents of urban centres such as Douala and Yaoundé [2], Ilorin [21], have a higher level of malaria awareness compared to rural centres [29]. This discrepancy on malaria knowledge between urban and rural settings reflects the differences in socioeconomic status, educational level and access to information. Literacy can affect the success of the malaria control program as the illiteracy level has also a direct correlation with the prevalence of disease along with other factors. With the high illiteracy rates, lack of formal education and geographic isolation, the information and communication delivery mechanism should be made accessible to these communities.

The main types of malaria preventive measures frequently reported by the current study participants include: avoiding stagnant water $70 \%$, clearing of bushes $61 \%$. This agrees with observations from Ethiopia [30] and Nigeria [2] but lower than that of Humphrey [21]. In the rural areas, the attribution by the respondents of physical, environmental and dietary factors as causative agents of malaria underscores the need for effective intervention to improve the level of knowledge in these communities. This is not surprising, because the message "environmental sanitation" is an important component of the National Malaria Control Programme and the key for local administration to maintain a clean environment. More than half of the respondents demonstrated a gap of knowledge on the real cause of malaria (26.8\% I did not know how the disease was transmitted, $28.8 \%$ had misconceptions) and that was similar to the findings of Humphrey [31]. The lack of knowledge about the role of mosquitoes in malaria transmission will jeopardize control measures if this is not addressed appropriately. Astonishingly, no currently recommended World Health Organization 
(WHO) interventions for malaria prevention such as IRS is implemented in the areas while LLINs distribution is inconsistent, sporadic and insufficient.

Shockingly, $47.2 \%$ of the studied population mentioned taking native concoction as prophylaxis to prevent the occurrence of malaria. These findings were higher than that of Oguonu (2005) who found out that $17 \%$ of caregivers in Nigeria would take native concoctions as malaria preventive measures whereas in Uganda [30] more than $70 \%$ of people with malaria did not seek care from the public health institutions [32]. This may be attributable to lack of education and communication by the local health authorities, the inaccessibility to mass media and difficulty to physically get access to public health institutions. Interventions strategies are yet to be implemented in these enclaved communities. The implementation of NightWatch strategies including the use of credible and popular local celebrities as messengers, distributing consistent messages across multiple concerts and public events in these remote areas over time will raise community awareness and mobilization in the fight against malaria.

It is estimated 136 million Insecticide Treated Nets (ITNs) were distributed to endemic countries in 2012 [33]. In Cameroon, promotion and periodic distribution of ITNs have been going on for more than 8 years. Government, private and non-governmental organizations have been involved in ITNs sourcing and distribution. More astonishing and less encouraging it is that, out of the 851 respondents, only $93(11.05 \%)$ possessed at least one mosquito bed net and they got it from the government during the first-ever mass distribution campaign in 2008. Household heads mentioned that a household member would receive a single ITN, but this is only when she had delivered a child. The ITNs use rate found in this study is considerably lower than $80 \%$ which is the targeted coverage of the Roll Back Malaria [7]. Moreover, these findings are similar to that of Krezanoski (2010) in Madagascar and lower than that observed by Mayala (2015) in Tanzanian and Musoke (2015) [34-36] in Uganda. Studies by Kimbi (2014b) [19] reported that $47 \%$ of respondents in rural Bolifamba used ITNs for protection against malaria while in urban centre like Yaoundé, Ndo [2] reported $69 \%$. The ownership of ITNs was significantly different among various communities $(P<.0 .001)$. This discrepancy may be due to poor state of roads leading to some communities such as Kitta Balue, Ngolo Metoko, and Kombe Balondo. The usage of nets was not significantly associated with education level $(\chi 2=7.533, p=0.110)$.

During fieldwork, bed net use was observed to be irregular. Some members of the household did not necessarily sleep under one, and the main constraint mentioned was heat it generates at night. Others stated that they use it for fishing because of the good mesh size, drying and packaging 'egusi' melon seeds (Colocynthis citrullus $L$.) for protection against rodents. Those not having ITNs (85.27\%) reported that, since their purchasing power is so limited, they cannot afford ITNs. Studies in other parts of Cameroon $[10,19,35]$ and elsewhere [37] have shown that decrease rates of malaria transmission and subsequent morbidity when ITNs/LLINs were widely distributed. One of the key component in the prevention and control of malaria at community level by the National Malaria Control Program is the selection and training of community health workers (CHWs). The CHWs can serve as a bridge between the public health authorities and the communities in the distribution of LLINs in order to make mosquito nets available to all members of the communities.

Four points zero four percent $4.04 \%$ reported to use door screens while window screens were used by $1.54 \%$ of respondents. These findings are in agreement with previous studies in rural as well as urban areas, which showed that people's use of door screens as their malaria preventive measures was still very low $[2,32]$. In the case of door screens, frequent opening of the door during entry and exit of household members limits this barrier method of preventing human-vector contact [32].

The number of respondents who claimed to have experienced any malaria episode $(21.0 \%)$ a week before the interview was phenomenal, considering that malaria is endemic in this area. In the present study, the proportion of malaria occurrence within three months showed that $39.9 \%$ of all the respondents have experienced the disease at least one time, which is a serious cause for alarm. These are comparable to findings in Tanzania where respondents had 1-2 episodes of malaria per year and over $71 \%$ did not attend to any farm activity [38]. Similarly, in Nigeria children had 0-5 episodes and adults had 0-1 episodes during a year [39]. The majority of people with symptomatic malaria episodes are treated at home because of inaccessibility to the community by road. This recurrent malaria episode experienced by participants could be attributed to frequent exposure to infective mosquito bites, absence of preventive measures or respondents may have considered every other fever as malaria. The proximity to the thick equatorial forest also provides appropriate breeding sites for malaria vectors.

This study had three main limitations. First, our data and conclusions are based on participant responses, and therefore reflect what participants have told us, and cannot be verified. Secondly, this study lacks data on participant's sources of malaria information and lastly health-seeking behavior of the head of households was not ascertained. The physical inaccessibility of some villages due to the very poor state of roads was a serious constraint in this study [40-42].

\section{Conclusions}

This study showed that most people had fair knowledge about malaria prevention and control. Despite this fair knowledge and good attitudes, practices towards malaria prevention and control were very poor. The level of knowledge on the transmission of malaria and the level of ownership of ITNs as a preventive measure against malaria was very low among the household members in the study population. The present study also showed that a considerable proportion of respondents had misconceptions about the cause and transmission of malaria. In order to close the gap between knowledge about transmission and ownership and use of bed nets as a preventive measure, the CHWs activities need to be re-energized and Night Watch concept implemented to out-of-reach, rural and remote communities. Such interventions will aim at improving the knowledge of rural dwellers regarding malaria and should involve the active participation of members of the community, traditional healers, and more importantly should be sustainable.

\section{Acknowledgments}

We want to acknowledge University of Buea for giving any support required to carry out this research. We thank the participants from the communities who made this study possible and the village Chiefs who assisted with this work. We would also like to express our appreciation to Betombo Tabitha Ongle, a health worker who serves as a translator during the study period. Our deepest appreciation goes to Mr. Kamadje Albert for his unlimited and financial support.

\section{Conflicts of Interest Statement}

The authors have no conflicts of interest concerning the work reported in this paper

1. Department of Microbiology and Parasitology, University of Buea, P.O.Box 63 Buea, South West Region, Cameroon. Tel: (237) 6745347 22, E-mail: tientcheb@yahoo.com

2. Department of Microbiology and Parasitology, University of Buea, P.O.Box 63 Buea, South West Region, Cameroon.Tel: (237) 6760338 46, Email:Jerome1.frucho@gmail.com

3. Department of Microbiology and Parasitology, University of Buea, P.O.Box 63 Buea, South West Region, Cameroon.Tel: (237) 6777348 16, Email: damian.anong@ubuea.cm

4. Department of Public Health, Faculty of Health Science. Tel:(237) 67039 5424 Email:tiigou4@yahoo.com 
5. Department of Microbiology and Parasitology, University of Buea, P.O.Box 63 Buea, South West Region, Cameroon. Tel: (237) 6777707 98, E-mail: wifon@yahoo.com

\section{Authors' Contributions}

BT participated in the design of the study, in data collection and the writeup of the manuscript. JFC participated in the design and data collection. DNA conceived the study, participated in the design and coordination, and revision of the manuscript. $\mathrm{HN}$ participated in data analysis and revision of the manuscript. TKN conceived the study, participated in the design, the write-up of the manuscript and revision of the manuscript. All authors read and approved the final manuscript.

\section{References}

1. Antonio-Nkondjio Christophe, Jean Atangana, Cyrille Ndo, and Parfait Awono-Ambene, et al. "Malaria transmission and rice cultivation in Lagdo, Northern Cameroon." Transactions of the Royal Society of Tropical Medicine and Hygiene102 (2008): 352-359.

2. Ndo Cyrille, Benjamin Menze-Djantio, and Christophe Antonio-Nkondjio. "Awareness, attitudes and prevention of malaria in the cities of Douala and Yaoundé (Cameroon)." Parasites and Vectors 4 (2011).

3. Sayang Collins, Mathieu Gausseres, Nicole Vernazza-Li, and Denis Malvy, et al. "Treatment of malaria from monotherapy to artemisinin-based combination therapy by health professionals in urban health facilities in Yaoundé, central province, Cameroon." Malaria Journal 8 (2009): 176.

4. National Malaria Control Programme. Regional Malaria Control Unit, South West Region. Annual Report of Activities for the year (2010).

5. Minsanté Cameroun. "Ministry of Public Health: National strategic plan to fight against malaria 2011-2015 Cameroon: Ministry of Public Health" 2012.

6. World Health Organization: The Global Strategic Plan 2005-2015-Roll Back Malaria. Geneva (2010).

7. Ali Innocent M, Palmer M Netongo, Barbara Atogho-Tiedeu, and Eric-Olivier Ngongang et al. "Amodiaquine-Artesunate versus Artemether-Lumefantrine against Uncomplicated Malaria in children less than 14 years in Ngaoundere, North Cameroon: efficacy, safety, and baseline drug resistant mutations in pfcrt, pfmdr1, and pfdhfr genes." Malar Res Treat 6 (2013).

8. Kimbi Helen Kuokuo, Irene Ule Ngole Sumbele, Malaika Nweboh, and Judith $\mathrm{K}$ Anchang-Kimbi, et al. "Malaria and haematologic parameters of pupils at different altitudes along the slope of Mount Cameroon: a cross-sectional study." Malar J 12 (2013):193.

9. Tchinda Viviane Hélène Matong, Antoine Socpa, Aubin Armand Keundo, and Francis Zeukeng, et al. "Factors associated to bed net use in Cameroon: a retrospective study in Mfou health district in the Centre Region." Pan Afr Med J 12 (2012):112.

10. Etang Josiane, Philippe Nwane, Jean Arthur Mbida, and Michael Piameu, et al. "Variations of insecticide residual bio-efficacy on different types of walls: results from a community-based trial in south Cameroon." Malar J 10 (2011): 33.

11. Einterz Ellen M. "Perceptions of malaria transmission, presentation and management in northern Cameroon. "Trans R Soc Trop Med Hyg 97 (2003): 51-52.

12. Nkuo-Akenji Theresa K, Nelson N Ntonifor, Maze B Ndukum, and Helen K $\mathrm{Kimbi}$, et al. "Environmental factors affecting malaria parasite prevalence in rural Bolifamba, South- West Cameroon." Afr J Health Sci 13 (2006): 40-46.

13. Kimbi Helen Kuokuo, Sarah Bi Nkesa, Judith Lum Ndamukong-Nyanga, and Irene Ule Ngole Sumbele, et al. "Knowledge and perceptions towards malaria prevention among vulnerable groups in the Buea Health District, Cameroon." BMC Public Health (2014a): 883.

14. Bauch Julie A, Jessica J Gu, Mwinyi Msellem and Andreas Martensson, et al. "Perception of malaria risk in a setting of reduced malaria transmission: a qualitative study in Zanzibar." Mal J (2013): 75.

15. Laar Alexander Suuk, Amos Kankponang Laar, Philip Ayizem Dalinjong Laar.
"Community perception of malaria and its influence on health-seeking behaviour in rural Ghana: a descriptive study." Malaria World Journal (2013): 1.

16. Nkuo-Akenji Theresa K, Ntonifor N Ntonifor, Ching JK and Kimbi HK, et al. "Evaluating a malaria intervention strategy using knowledge, practices and coverage surveys in rural Bolifamba, southwest Cameroon." Transactions of the Royal Society of Tropical Medicine and Hygiene (2005): 325-332.

17. Third general census of population and housing. Third general population and housing census Cameroon. Presentation report of the final results, Republic of Cameroon (2010): 1-65.

18. Kimbi Helen Kuokuo, Sarah Bi Nkesa, Judith Lum Ndamukong-Nyanga and Irene Ule Ngole Sumbele, et al. "Socio-demographic factors influencing the ownership and utilization of insecticide-treated bed nets among malaria vulnerable groups in the Buea Health District Cameroon." BMC Research Notes (2014b): 624.

19. Syed Masud Ahmed, Rashidul Haque, Ubydul Haque, Awlad Hossain. "Knowledge on the transmission, prevention and treatment of malaria among two endemic populations of Bangladesh and their health-seeking behaviour." Mal J (2009): 173.

20. Obembe Abiodun, Adedayo Oduola, Kehinde Popoola, Adeyemi Ajao. "Community knowledge and practices as regards malaria in llorin City: implications for the elimination plan of the National Malaria Elimination Program." Asian Pacific Journal of Tropical Disease (2014): S583-S58.

21. Serengbe Gustave Bobossi, Jean-Methode Moyen, Rosine Fioboy and Edith Narcisse Beyam, et al. "Knowledge and perceptions about malaria in communities in four districts of the Central African Republic." BMC Research Notes (2015): 162.

22. Eyobo Margaret B, Adwok C Awur, Gregory Wani and Ahmed I Julla, et al. Malaria indicator survey 2009, South Sudan: baseline results at household level. Mal J (2014): 45.

23. Nsagha Dickson Shey, Anna Longdoh Njunda, Henri Lucien Fouamno Kamga and Jules Clement Nguedia Assob, et al. "Knowledge and practices relating to malaria in Ndu community of Cameroon: Signs and symptoms, causes and prevention." Journal of Public Health and Epidemiology (2011): 294-300.

24. Adedotun AA, Morenikeji OA, Odaibo AB. "Knowledge, attitudes and practices about malaria in an urban community in South-Western Nigeria". $J$ Vector Borne Dis (2010): 155-159.

25. Aderaw Zewdie and Gedefaw Molla. Knowledge, attitude and practice of the community towards malaria prevention and control options in anti-malaria Association Intervention Zones of Amahara National Regional State, Ethiopia. J Trop Dis (2013): 118.

26. Adebayo Ayodeji $M$, Oluwaseun $O$ Akinyemi and Eniola $O$ Cadmus. "Knowledge of malaria prevention among pregnant women and female caregivers of under-five children in rural southwest Nigeria". Peer Journal (2015): e792.

27. Abate Andargie, Abraham Degarege and Berhanu Erko. "Community knowledge, attitude and practice about malaria in a low endemic setting of Shewa Robit Town, northeastern Ethiopia." BMC Public Health (2013): 312.

28. Ouattara Allassane F, Giovanna Raso, Constant VA Edi and Jurg Utzinger, et al. "Malaria knowledge and long-lasting insecticidal net use in rural communities of central Côte d'Ivoire." Malaria Journal (2011): 288.

29. Tagbo Oguonu, Uche Okafor, Herbert Anayo Obu. "Caregivers's knowledge attitude and practice on childhood malaria and treatment in urban and rura communities in Enugu, south-east Nigeria." Public Health (2005): 409-414.

30. Nuwaha Fred. "People's perception of malaria in Mbarara, Uganda." Tropical Medicine and International Health (2002): 462-470.

31. World Health Organization, WHO Global Malaria Programme. World Malaria Report 2013, Geneva, Switzerland (2013).

32. Krezanoski Paul J, Alison B Comfort and Davidson H Hamer. "Effect of incentives on insecticide-treated bed net use in sub-Saharan Africa: a cluster randomized trial in Madagascar." Mal J (2010): 186.

33. Mayala Benjamin K, Carolyn A Fahey, Dorothy Wei and Maria M Zinga, et al. "Knowledge, perception and practices about malaria, climate change, livelihoods and food security among rural communities of central Tanzania." Infectious Diseases of Poverty (2015): 2. 
34. Musoke David, George Miiro, George Karani and Keith Morris, et al. "Promising Perceptions, Divergent Practices and Barriers to Integrated Malaria Prevent WHO ion in Wakiso District, Uganda: A Mixed Methods Study." PLOS ONE (2015): e0122699.

35. Apinjoh Tobias $\mathrm{O}$, Judith $\mathrm{K}$ Anchang-Kimbi, Regina $\mathrm{N}$ Mugri and Delphine A, et al. "The Effect of Insecticide Treated Nets (ITNs) on Plasmodium falciparum Infection in Rural and Semi-Urban Communities in the South West Region of Cameroon." PLOS ONE (2015): 1-13.

36. Atieli Harrysone E, Guofa Zhou, Yaw Afrane and Ming-Chieh Lee, et al. Ownership, usage, and malaria transmission in the highlands of western Kenya. Parasites \& Vectors (2011): 113.

37. Leonard E G Mboera, Elizabeth H Shayo, Kesheni P Senkoro and Susan $F$ Rumisha, et al. "Knowledge, perceptions and practices of farming communities on linkages between malaria and agriculture in Mvomero District, Tanzania." Acta Tropica (2010): 139-44.

38. Olalekan Musa Salihu, Nurudeen Ayodeji Sanni. "Malaria burden and the effectiveness of malaria control measures in Nigeria: a case study of Asa Local Government Area of Kwara State." Journal Economic and Sustainable Developmeny (2013): 295-308.
39. Kometa Sunday Shende, Jude Ndzifon Kimengsi. "Transport as an Impediment to an Economically Endowed Region: The Case of Ekondo - Titi Sub Division of Cameroon." Greener Journal of Environmental Management and Public Safety (2013): 032-039.

40. Legesse M, Deressa W. "Community awareness about malaria, its treatment and mosquito vector in rural highlands of central Ethiopia." Ethiopia Journal Health Development (2009): 40-47.

41. World Health Organization. WHO Global Malaria Programme. World Malaria Report 2013, Geneva, Switzerland (2013).

42. World Health Organization. WHO Global Malaria Programme World Malaria Report 2014, Geneva, Switzerland (2017)

How to cite this article: Bonaventure Tientche, Jerome Fru-Cho, Damian Nota Anong and Hermann Ngouakam, et al. "Assessment of Knowledge, Perception and Practices about Malaria in Some Rural Communities of South West Region, Cameroon". J Health Med Informat 11 (2020) doi: 10.37421 jhmi.2020.11.344 